\title{
Estudo das indicações de cesariana em uma maternidade de referência em baixo
}

\section{risco}

\author{
Study of cesarean section indications in a low-risk referral maternity hospital \\ Estudio de las indicaciones de cesárea en una maternidad de referencia de bajo riesgo
}

\section{Resumo}

Objetivo: Analisar se as indicações de operação cesariana realizadas em maternidade de referência em baixo risco de Sergipe estão de acordo com a diretriz do Ministério da Saúde publicada em 2016. Métodos: Estudo documental, prospectivo, qualitativo, descritivo e de corte transversal, com coleta de dados através da análise de 318 prontuários médicos de puérperas pós cesárea no período de 2018 a 2019. Os dados obtidos foram analisados por estatística descritiva com auxílio do software Graph Pad Prism e SPSS 20. O estudo foi aprovado por Comitê de Ética em Pesquisa. Resultados: A principal indicação encontrada foi uma operação cesariana anterior, seguidas de outras como: desproporção céfalo-pélvica, apresentação pélvica, iteratividade, sofrimento fetal, macrossomia, falha de indução e pós-datismo. Conclusão: Conforme a principal indicação encontrada, as indicações não estão totalmente de acordo com a diretriz. Entretanto, outras indicações encontradas estão corretas, porém fogem da diretriz por serem situações de cuidado intra-parto e de urgência, não discutidas no documento.

Palavras-chave: Cesárea; Obstetrícia; Saúde da mulher.

\begin{abstract}
Objective: To analyze whether the indications for cesarean section performed in a low risk reference maternity in Sergipe are in accordance with the Ministry of Health guideline published in 2016. Methods: Documentary, prospective, qualitative, descriptive and cross-sectional study, with data collection through analysis of 318 postcesarean section medical records from 2018 to 2019. The data obtained were analyzed using descriptive statistics with the aid of the Graph Pad Prism and SPSS 20 software. The study was approved by the Research Ethics Committee. Results: The main indication found was a previous cesarean section, followed by others such as: cephalopelvic disproportion, breech presentation, iterativity, fetal distress, macrosomia, induction failure and post-datism. Conclusion: According to the main indication found, the indications are not fully in accordance with the guideline. However, other indications found are correct, but are outside the guideline because they are situations of intradelivery care and urgency, not discussed in the document.
\end{abstract}

Keywords: Cesarean section; Obstetrics; Women's health. 


\section{Resumen}

Objetivo: Analizar si las indicaciones de cesárea realizadas en una maternidad de referencia de bajo riesgo en Sergipe se ajustan a la directriz del Ministerio de Sanidad publicada en 2016. Métodos: Estudio documental, prospectivo, cualitativo, descriptivo y transversal con recogida de datos mediante análisis de 318 historias clínicas poscesáreas de 2018 a 2019. Los datos obtenidos se analizaron mediante estadística descriptiva con la ayuda del software Graph Pad Prism y SPSS 20. El estudio fue aprobado por el Comité de Ética en Investigación. Resultados: La principal indicación encontrada fue una cesárea previa, seguida de otras como: desproporción cefalopélvica, presentación de nalgas, iteratividad, sufrimiento fetal, macrosomía, falla de inducción y posdatismo. Conclusión: De acuerdo con la indicación principal encontrada, las indicaciones no están completamente de acuerdo con la guía. Sin embargo, otras indicaciones encontradas son correctas, pero están fuera de la guía por tratarse de situaciones de atención y urgencia intraparto, no discutidas en el documento.

Palabras clave: Cesárea; Obstetricia; Salud de la mujer.

\section{Introdução}

A cesariana é uma das cirurgias mais comuns no mundo atual e principalmente no Brasil, líder mundial desse tipo de parto. Segundo Ribeiro (2016), a operação cesariana no país apresentou uma progressão gradativa desde os anos 70 quando as taxas eram próximas de $15 \%$ com crescimento exagerado e frequente a partir dos anos 2000 , chegando a cerca de $56 \%$ na atualidade. Além disso, há uma diferença significativa entre os serviços públicos (40\%) e os privados de saúde (85\%) (MS,2016).

Quando realizada em decorrência de razões médicas, a operação cesariana pode ser benéfica tanto do ponto de vista materno quanto do perinatal. Entretanto, é frequentemente utilizada de forma desnecessária, sem justificativa para as altas taxas observadas (Amorim; Souza \& Porto, 2010). Estudos mostram que até um determinado patamar de indicações, a mortalidade materno-fetal diminui ao englobar pacientes que necessitam realmente da cesariana; e, a partir de determinado valor, pode haver até uma piora nesses indicadores de saúde (Xie R., et al., 2015).

Para Carlos \& Travers(2016), a taxa de mortalidade materna e neonatal da operação cesariana é de quatro a cinco vezes maior em relação aos partos vaginais, particularmente nas cesáreas eletivas. Há ainda aumento do risco de morbidade perinatal, sobretudo de admissão na unidade de terapia intensiva neonatal e síndrome da angústia respiratória do recémnascido, que podem ser reduzidas se a cesariana eletiva for realizada acima da $39^{\mathrm{a}}$ semana de gravidez.

Por outro lado, outros estudos defendem que o aumento das taxas não está relacionada com aumento de mortalidade materna, o que permitiria alargar as indicações dessa cirurgia, permitindo-se, até mesmo, realizar a pedido da paciente (O’Dwyer, et al., 2012). Embora, como qualquer outra cirurgia, não esteja isenta de complicações anestésicas e operatórias e problemas relacionados à transfusão de sangue(Weidle, et al., 2014).

São complexas as causas do uso excessivo da cesariana no Brasil. Essas, incluem a maneira como a assistência ao nascimento é organizada, ainda bastante centrada na atuação individual dos profissionais em contraposição à abordagem multidisciplinar e de equipe; as características socioculturais, as características da assistência pré-natal, que comumente deixa de preparar adequadamente as mulheres para o parto e a qualidade dos serviços que assistem os nascimentos, assim como a alta demanda e dificuldade de assistir a todas da maneira que se deveria.

Segundo a Organização Mundial de Saúde (WHO,2015), desde 1985, a comunidade médica internacional considera que a taxa ideal de cesáreas fique entre 10 e 15\%, recomendação que surgiu de uma declaração feita por um grupo de especialistas em saúde reprodutiva durante uma reunião promovida pela OMS em Fortaleza, no Brasil, e que diz: "Não existe justificativa para qualquer região do mundo ter uma taxa de cesárea maior do que 10-15\%”. Porém, o grupo baseou essa afirmação em uma revisão dos poucos dados disponíveis na época, provenientes principalmente de países no norte da Europa, que mostravam ótimos resultados maternos e perinatais com essas taxas de cesárea. Como uma adaptação à realidade demográfica e cultural brasileira com auxílio de dados da pesquisa Nascer no Brasil (Lansky, et al., 2014) , uma taxa de referência para o país seria em torno de $25-30 \%$. 
Visto essa problemática, o Ministério da Saúde (MS), com o objetivo de tentar mudar essa realidade, realiza a implantação de importantes planos de ação. Como exemplo, há o projeto Parto Adequado, lançado em 2015 e que em 18 meses evitou a realização de 10 mil operações desnecessárias, evidenciando resultados transformacionais e reforçando a necessidade de empenho contínuo para impactar no atual cenário nacional (ANS, 2016).

Em março de 2016, o MS elaborou as " Diretrizes de Atenção à Gestante: a operação cesariana" considerando a necessidade de se estabelecerem parâmetros e um referencial para as condutas acerca da operação cesariana no Brasil e diretrizes nacionais para a sua utilização e acompanhamento das mulheres a ela submetidas. Foram realizadas diversas revisões sistemáticas com classificação e interpretação das evidências científicas; não foram abordadas situações que ocorrem no cuidado intraparto, nas urgências e no atendimento às gestantes com comorbidades cujas indicações dessa intervenção cirúrgica podem variar de acordo com a doença ou condição específica (MS,2016).

É importante oferecer orientações e recomendações baseadas em evidências científicas no intuito de promover e proteger a saúde e o bem-estar da mulher e da criança. Assim, este trabalho buscou pesquisar as indicações para a operação na maternidade de baixo risco Santa Isabel e analisar se estavam de acordo com a portaria realizada pelo MS.

\section{Metodologia}

Estudo documental, prospectivo, qualitativo, descritivo e de corte transversal, com coleta de dados através da análise dos prontuários médicos de puérperas no pós-parto cesárea disponíveis durante a internação das mesmas, sob declaração de manter compromisso e confidencialidade em relação a todas as informações obtidas relativas ao projeto. A população de estudo foi composta por 318 pacientes do Sistema Único de Saúde (SUS) atendidas na maternidade de baixo risco Santa Isabel localizada em Aracaju, Sergipe, no período de 2018 a 2019, com o intuito de avaliar se o procedimento da cesárea segue as Diretrizes de Indicação de Cesariana, proposta pelo Ministério da Saúde em 2016. O número de sujeitos utilizados nesta pesquisa foi considerado e confirmado pelo estatístico como sendo suficiente para atingir os objetivos deste trabalho.

O estudo estatístico utilizou Graph Pad Prism e SPSS 20. Em todos os testes, fixou-se nível de significância em 0,05 ou $5 \%$.

A pesquisa respeitou as normas da Declaração de Helsinque e da Resolução no 466/2012, do Conselho Nacional de Saúde, para pesquisa em seres humanos, com Termo de Consentimento Livre e Esclarecido; iniciou-se após a aprovação do Comitê de Ética em Pesquisa da Universidade Tiradentes de Aracaju.

\section{Resultados e Discussão}

Foram estudados 318 prontuários de operações cesarianas, cujas indicações mais prevalentes em ordem decrescente foram: uma césarea anterior, desproporção céfalo-pélvica, apresentação pélvica, iteratividade, sofrimento fetal, macrossomia, falha de indução e pós-datismo. Esses dados obtiveram significância estatística (p<0.05) e encontram-se na Figura 1 abaixo. 
Figura 1. Indicações de cesariana na Maternidade Santa Isabel em Aracaju, 2019.

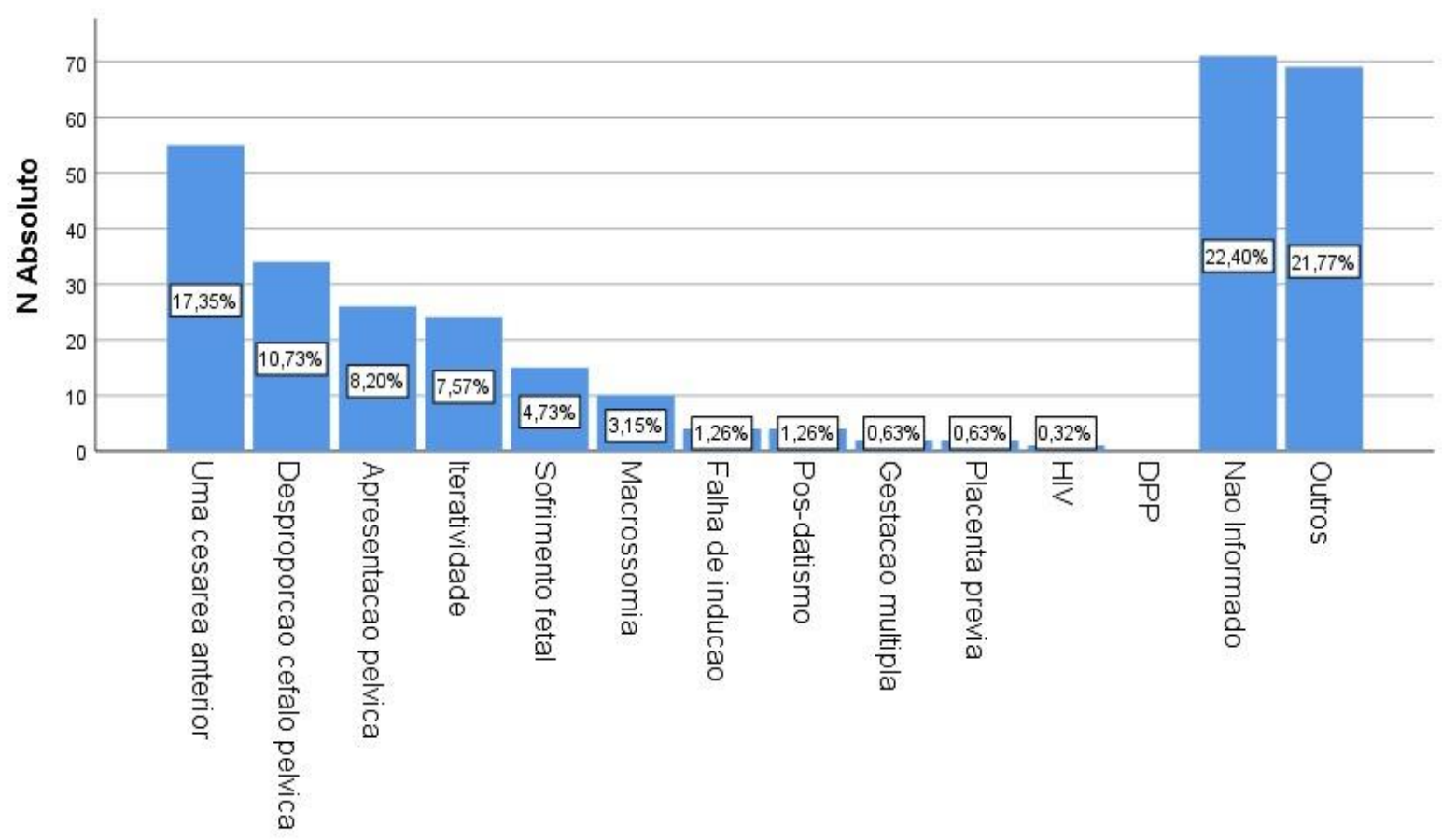

Fonte: Autores.

Como observado, a principal indicação de cesárea encontrada no estudo foi uma operação cesariana anterior. Pela diretriz do MS, é recomendado que as mulheres com operações cesarianas prévias sejam esclarecidas de que há um aumento no risco de ruptura uterina com o parto vaginal, a princípio, baixo, porém que aumenta à medida que aumenta o número de cesarianas prévias, porém, na ausência de outras contraindicações, é recomendado encorajar a tentativa de parto vaginal, mediante termo de consentimento informado. O aconselhamento sobre o modo de nascimento em gestantes com operação cesariana prévia deve considerar as preferências, os riscos e benefícios das vias de parto (MS,2016).

Em mulheres com operação cesariana anterior, a taxa de mortalidade materna é maior nas gestantes submetidas a uma nova cesariana programada quando comparadas àquelas que tiveram parto vaginal. Quanto à necessidade de hemotransfusão e ruptura uterina, o risco foi menor nas gestantes submetidas a uma nova operação cesariana (Guise, et al., 2010).

Dois estudos observacionais estudaram mulheres com cesariana prévia que tentaram parto vaginal e observaram uma taxa de sucesso de 74\%-80\% em um estudo (Cahill, et al., 2010) e 72\%-76\% no outro (Tahseen \& Griffiths, 2010). Comparando as mulheres com uma operação cesariana prévia que tiveram parto vaginal planejado e operação cesariana programada, independentemente da idade gestacional, é baixa a taxa de ruptura uterina e de infecção pós-parto. Não houve diferença significativa após duas ou mais operações cesarianas prévias quando comparadas com àquelas que tiveram apenas uma operação cesariana.

O risco de ruptura uterina é três a cinco vezes maior quando o trabalho de parto é induzido, por qualquer meio (Dekker, et al., 2010). Ademais, aumenta quando o intervalo entre os partos diminui (Kayem, et al., 2012), porém não contraindica o parto vaginal subsequente se as condições obstétricas forem favoráveis. As complicações neonatais em caso de mulheres com operação cesariana prévia que entraram em trabalho de parto e evoluíram para cesariana intraparto são maiores quando comparadas com aquelas que preferiram cesariana programada. Contudo, a prevalência dessas complicações é muito baixa. 
São condições que estão fortemente relacionadas a um parto vaginal após operação cesariana prévia: parto vaginal prévio (especialmente se ele ocorreu após uma cesariana prévia) e condições favoráveis do colo uterino (avaliado ou não através do escore de Bishop). Condições maternas como diabetes, obesidade, idade materna e multiparidade e necessidade de indução de trabalho de parto podem reduzir as taxas de parto vaginal, no entanto não o contraindicam (Haumonté, et al., 2012).

A iteratividade, significado dado a mulher que já teve duas ou mais cesáreas, caracterizou a quarta indicação mais encontrada, assim como uma das maiores indicações no estudo (Novo, et al., 2017). Em mulheres com duas operações cesarianas prévias a conduta deve ser individualizada e os profissionais e instituições de saúde devem ter resguardada sua autonomia em relação a aceitação ou não da assistência ao parto vaginal após duas operações. A cesariana é recomendação direta para mulheres com três ou mais cesarianas prévias e mulheres com cicatriz uterina longitudinal de operação cesariana anterior (MS,2016).

As pesquisas de Pádua, et al. (2010) e Novo, et al. (2017) abordaram a indicação de cesárea por desproporção céfalopélvico (DCP), sendo que na primeira a indicação foi fora de trabalho de parto e na segunda intraparto. No presente estudo, DCP foi a segunda indicação mais encontrada, diagnosticada no intraparto. Amorim, Souza \& Porto (2010) dizem que a DCP frequentemente é diagnosticada de forma inadequada pois este só pode ser realizado durante o trabalho de parto, baseado nos dados de sua evolução, conforme registro no partograma, não devendo se supor uma DCP, mesmo quando se conhece os principais fatores de riscos. Inclusive, segundo a diretriz, inexiste evidência que apoie o uso da pelvimetria clínica ou da radiografia para predizer a ocorrência de falha de progressão do trabalho de parto (MS,2016).

A obesidade materna foi um indicador de cesárea encontrado nos artigos de Pádua, et al (2010) e Oliveira, et al. (2016), porém não foi encontrada no presente estudo como indicação direta da operação e a diretriz brasileira e outras evidências científicas não apoiam a indicação de cesariana. Para obesas mórbidas, a decisão deve ser individualizada (Homer, et al., 2011).

Novo, et al. (2017) trazem que apresentação pélvica também é uma indicação de cesárea no país, sendo encontrada como terceira maior indicação no presente estudo. A diretriz londrina, NICE, que serviu de base para o Ministério da Saúde, indica a versão cefálica externa como uma opção para fetos em apresentação pélvica antes da cesariana e é recomendada a partir de 36 semanas de idade gestacional, mediante termo de consentimento informado e na ausência de contraindicações como cesariana prévia ou trabalho de parto.

Entretanto, avaliando o efeito do modo de nascimento em gestações a termo com apresentação pélvica, uma revisão sistemática mostrou redução de mortalidade perinatal e neonatal assim como morbidade neonatal grave quando se realizou uma operação cesariana, recomendada a partir de 39 semanas de gestação (MS,2016). O risco foi de 1,6\% no grupo da operação cesariana programada e de $5 \%$ no do parto vaginal planejado. Assim, a recomendação oficial é a operação cesariana a partir de 39 semanas de idade gestacional, sugerindo-se aguardar o início do trabalho de parto. Caso a mulher decidir por um parto pélvico vaginal, é recomendado que tenha o termo de consentimento livre esclarecido sobre o maior risco de morbidade e de mortalidade e que a assistência seja realizada por profissionais experientes na assistência ao parto pélvico.

Sofrimento fetal agudo (SFA) foi apontado como indicação de cesárea tanto no presente estudo como também no estudo Novo, et al. (2017). O SFA é conceitualmente a asfixia fetal persistente e progressiva cuja gravidade, se não revertida, leva ao esgotamento dos mecanismos compensatórios, resultando em morte celular neuronal, podendo ser reconhecido de forma indireta por alterações nas atividades biofísicas, principalmente em sua frequência cardíaca, identificada pela ausculta ou traçado cardiotocográfico (CTG). Como a diretriz não abordou situações de cuidado intraparto de urgência, essa indicação não foi discutida (Montenegro C \& Rezende J 2014).

A cesárea indicada por pós-datismo, gestação com duração igual ou superior a 40 semanas, encontrada como indicação em poucos casos no presente estudo, também foi encontrada no artigo Novo, et al. (2017) e não é recomendada como 
indicação para cesariana na diretriz brasileira. Em manual do Ministério da Saúde sobre atenção ao pré-natal de baixo risco de 2012, a conduta de induzir o trabalho de parto em todas as gestantes com 41 semanas de gravidez é preferível à avaliação seriada do bem-estar fetal, pois se observou menor risco de morte neonatal e perinatal e menor chance de cesariana no grupo submetido à indução do parto com 41 semanas.

Assim como encontrado nos resultados desta pesquisa, Novo, et al. (2017) trazem que a falha de indução foi uma outra indicação de cesárea. A indução do parto consiste em estimular artificialmente as contrações uterinas coordenadas e efetivas antes de seu início espontâneo, levando ao desencadeamento do trabalho de parto em mulheres a partir da $22^{\mathrm{a}}$ semana de gravidez. As principais indicações para indução do trabalho de parto, abrange desde indicações eletivas, como gestantes na $41^{\mathrm{a}}$ semana de gravidez sem doença obstétrica, até quando a interrupção da gravidez se faz de fato necessária devido a doenças maternas ou fetais, que fazem com que o prolongamento da gestação ocasione risco de morte materno ou fetal (Souza, et al., 2010). Para Sampaio, et al. (2004), na maioria das condições clínicas representativas de indicação para indução do parto, a cérvice desfavorável é encontrada, o que pode frequentemente acarretar falha da indução e consequente aumento da incidência de cesarianas.

Outras recomendações pela diretriz para indicação são: gestação múltipla, placenta prévia, acretismo placentário, infecção pelo vírus HIV, hepatite C quando há coinfecção com HIV e infecção por herpes vírus simples.

Neste estudo, foi observado apenas três casos com indicação por gestação múltipla, em dois o primeiro feto tinha apresentação pélvica, um caso com indicação de placenta prévia e outro por infecção pelo vírus HIV. Acretismo placentário, coinfecção de hepatite C com HIV e infecção por herpes vírus simples não foram encontrados. Esses resultados são explicados pela baixa incidência dessas situações na maternidade, referência em baixo risco.

Em relação à gestação múltipla, o TwinBirthStudy, um grande estudo randomizado no NICE comparou operação cesariana programada versus parto vaginal planejado e concluiu que, em gestações gemelares entre 32 semanas e 38 semanas e 6 dias de idade gestacional, a operação cesariana programada não diminuiu nem aumentou o risco de morte fetal ou neonatal, ou de morbidade neonatal grave (Barret JF, et al., 2013). Assim, diferentes abordagens são possíveis e podem ser necessárias de acordo com as características da gestação, principalmente a corionicidade e a presença ou não de comorbidades. A determinação do momento e modo de nascimento deve ser individualizada. No caso de gestação gemelar não complicada cujo primeiro feto tenha apresentação não cefálica, a operação cesariana é recomendada.

Quanto maior o número de cesarianas anteriores, maior o risco de placenta prévia e de acretismo placentário (duas cesarianas prévias trazem um risco de $23-40 \%$ enquanto três ou mais, de 35-67\% em uma próxima gestação) (Guise, et al., 2010).

Sobre a placenta prévia, classicamente as formas centro-total ou centro-parcial têm sido definidas como indicação de operação cesariana, uma vez que a placenta oclui total ou parcialmente o canal cervical no momento do parto e é evidente o risco de hemorragia e morte fetal caso o trabalho de parto progrida nessas circunstâncias, podendo ocorrer o óbito materno. Sugere-se programar a operação cesariana eletiva a partir de 37 semanas de acordo com cada caso. Os demais tipos de placenta prévia (inserção baixa ou marginal) não se constituem por si só indicações para realização desta via de parto (MS,2016).

Em gestantes com placenta prévia é recomendada a realização de um exame ultrassonográfico com Doppler entre 28 e 32 semanas de idade gestacional, ou antes, se possível, para investigação de acretismo placentário. As gestantes com esse diagnóstico devem receber atenção especializada em serviços obstétricos de referência e programação da cesariana. Nas situações de suspeita de placenta increta ou percreta, pondera-se programar para ser realizada entre 34 e 36 semanas de gestação com a presença de dois obstetras experientes, anestesista e pediatra, bem como equipe cirúrgica de retaguarda, tipagem sanguínea e a reserva de hemocomponentes para eventual necessidade durante o procedimento em uma maternidade de alto risco (MS,2016). 
Em situações de infecções, as recomendações para gestantes com HIV/AIDS seguem as orientações do Protocolo Clínico e Diretrizes Terapêuticas: Prevenção da Transmissão Vertical de HIV, Sífilis e Hepatites Virais. Em mulheres com carga viral desconhecida ou maior que 1.000 cópias $/ \mathrm{ml}$ após 34 semanas de gestação, a operação cesariana eletiva na $38^{\circ}$ semana de gestação diminui o risco de transmissão vertical, a fim de evitar o trabalho de parto e a ruptura prematura das membranas. Para gestantes em uso de antirretroviral e com supressão da carga viral sustentada, caso não haja indicação de cesariana por outro motivo, a via de parto vaginal é indicada(Calvert \& Ronsmans, 2013).Caso a gestante com indicação de operação cesariana eletiva inicie o trabalho de parto antes da data prevista para a intervenção cirúrgica e chegue à maternidade com dilatação cervical mínima (menor que $4 \mathrm{~cm}$ ), o obstetra deve iniciar a infusão intravenosa de AZT e realizar a operação cesariana, se possível após 3 horas da infusão. Sempre que possível, proceder ao parto empelicado (MS,2016).

Infecção por hepatite $\mathrm{C}$ só indica operação cesariana programada diretamente se coinfecção com HIV.

Quanto ao vírus Herpes, os estudos são de baixa qualidade, porém acredita-se haver plausibilidade biológica para que se considere a cesárea como capaz de contribuir para a redução da transmissão vertical em gestantes com infecção primária ativa no último trimestre ou com infecção herpética ativa (primária ou secundária) no momento do parto (MS,2016).

\section{Considerações Finais}

O estudo revelou que a principal indicação de operação cesariana na maternidade foi de uma cesárea anterior, o que não está de acordo com as indicações da diretriz do Ministério da Saúde pois deveria ser realizada a tentativa do parto vaginal. Entretanto, outras indicações encontradas como apresentação pélvica e iterativade estão de acordo assim como indicações que acontecem no cuidado intra-parto e de urgência como desproporção céfalo-pélvica, porém, estas são situações que não são abordadas na diretriz.

Novos estudos devem ser realizados nas maternidades com o objetivo de analisar das indicações cesarianas para posteriormente haver uma modificação nestas e tentar, assim, diminuir os índices de partos operatórios.

\section{Nota}

Este trabalho foi financiado pela Fundação de Apoio à Pesquisa e Inovação Tecnológica do Estado de Sergipe (FAPITEC-SE).

\section{Referências}

Agência Nacional de Saúde Suplementar. (2016). Cartilha nova organização do cuidado ao parto e nascimento para melhores resultados de saúde: Projeto Parto Adequado-fase 1.

Amorim, M. M. R., Souza, A. S. R., \& Porto, A. M. F. (2010). Indicações de cesariana baseadas em evidências: parte I. Femina.

Barrett, J. F., Hannah, M. E., Hutton, E. K., Willan, A. R., Allen, A. C., Armson, B. A., \& Ross, S. (2013). A randomized trial of planned cesarean or vaginal delivery for twin pregnancy. New England Journal of Medicine, 369(14), 1295-1305.

Cahill, A., Tuuli, M., Odibo, A. O., Stamilio, D. M., \& Macones, G. A. (2010). Vaginal birth after caesarean for women with three or more prior caesareans: assessing safety and success. BJOG: An International Journal of Obstetrics \& Gynaecology, 117(4), 422-428.

Calvert, C., \& Ronsmans, C. (2013). HIV and the risk of direct obstetric complications: a systematic review and meta-analysis. PloS one, 8(10), e74848.

Carlo, W. A., \& Travers, C. P. (2016). Maternal and neonatal mortality: time to act. Jornal de pediatria, 92(6), 543-545.

Dekker, G. A., Chan, A., Luke, C. G., Priest, K., Riley, M., Halliday, J., \& Cull, V. (2010). Risk of uterine rupture in Australian women attempting vaginal birth after one prior caesarean section: a retrospective population-based cohort study. BJOG: An International Journal of Obstetrics \& Gynaecology, 117(11), 1358-1365.

Guise, J. M., Denman, M. A., Emeis, C., Marshall, N., Walker, M., Fu, R., \& McDonagh, M. (2010). Vaginal birth after cesarean: new insights on maternal and neonatal outcomes. Obstetrics \& Gynecology, 115(6), 1267-1278.

Haumonté, J. B., Raylet, M., Sabiani, L., Franké, O., Bretelle, F., Boubli, L., \& d'Ercole, C. (2012). Quels facteurs influencent la voie d'accouchement en cas de tentative de voie basse sur utérus cicatriciel? Journal de gynécologie obstétrique et biologie de la reproduction, 41(8), 735-752. 
Homer, C. S. E., Kurinczuk, J. J., Spark, P., Brocklehurst, P., \& Knight, M. (2011). Planned vaginal delivery or planned caesarean delivery in women with extreme obesity. BJOG: An International Journal of Obstetrics \& Gynaecology, 118(4), 480-487.

Kayem, G., Raiffort, C., Legardeur, H., Gavard, L., Mandelbrot, L., \& Girard, G. (2012). Critères d'acceptation de la voie vaginale selon les caractéristiques de la cicatrice utérine. Journal de gynécologie obstétrique et biologie de la reproduction, 41(8), 753-771.

Lansky, S., Friche, A. A. D. L., Silva, A. A. M. D., Campos, D., Bittencourt, S. D. D. A., Carvalho, M. L. D., \& Cunha, A. J. L. A. D. (2014). Pesquisa Nascer no Brasil: perfil da mortalidade neonatal e avaliação da assistência à gestante e ao recém-nascido. Cadernos de Saúde Pública, 30, S192-S207.

Ministério da Saúde, Brasil. (2012). Atenção ao pré-natal de baixo risco. http://bvsms.saude.gov.br/bvs/publicacoes/atencao_pre_natal_baixo_risco.pdf.

Ministério da Saúde, Brasil. (2016). Portaria No306, de 28 de março de 2016: Diretrizes de Atenção à Gestante: a operação cesariana.

Montenegro, C. B., \& de Rezende Filho, J. F. (2014). Obstetrícia fundamental. Rio de Janeiro: Guanabara Koogan.

National Institute for Health and Care Excellence (UK). (2017). Intrapartum care for healthy women and babies. (NICE Clinical Guidelines, No. 190.) https://www.nice.org.uk/guidance/cg190/resources/intrapartum-care-for-healthy-women-and-babies-pdf-351098664477557.

National Institute for Health and Care Excellence (UK). (2019). Caesarean section. (NICE Clinical Guidelines, No. 132.) https://www.ncbi.nlm.nih.gov/books/NBK552671/.

Novo, J. L. V. G., Pellicciari, C. R., de Arruda Camargo, L., Bálsamo, S. B., \& Novo, N. F. (2017). Indicações de partos cesáreos em hospitais de atendimento ao Sistema Único de Saúde: baixo e alto riscos. Revista da Faculdade de Ciências Médicas de Sorocaba, 19(2), 67-71.

O'Dwyer, V., Hogan, J. L., Farah, N., Kennelly, M. M., Fitzpatrick, C., \& Turner, M. J. (2012). Maternal mortality and the rising cesarean rate. International Journal of Gynecology \& Obstetrics, 116(2), 162-164.

Oliveira, R. R. D., Melo, E. C., Novaes, E. S., Ferracioli, P. L. R. V., \& Mathias, T. A. D. F. (2016). Factors associated to caesarean delivery in public and private health care systems. Revista da Escola de Enfermagem da USP, 50(5), 733-740.

Pádua, K. S. D., Osis, M. J. D., Faúndes, A., Barbosa, A. H., \& Moraes Filho, O. B. (2010). Fatores associados à realização de cesariana em hospitais brasileiros. Revista de Saúde Pública, 44, 70-79.

Pereira, A. S. et al. (2018). Metodologia da pesquisa científica. [e-book]. Santa Maria. Ed. UAB/NTE/UFSM. https://repositorio.ufsm.br/bitstream/handle/1/15824/Lic_Computacao_Metodologia-Pesquisa-Cientifica.pdf?sequence=1.

Ribeiro, L. B. (2016). Nascer em Belo Horizonte: cesarianas desnecessárias e prematuridade. Dissertação (Mestrado em Enfermagem) - Escola de Enfermagem da Universidade Federal de Minas Gerais, Belo Horizonte.

Sampaio, Z. S., Alencar Júnior, C. A., Feitosa, F. E. D. L., \& Amorim, M. M. R. D. (2004). Fatores associados ao parto vaginal em gestantes de alto risco submetidas à indução do parto com misoprostol. Revista Brasileira de Ginecologia e Obstetrícia, 26(1), 21-29.

Souza, A. S. R., Costa, A. A. R., Coutinho, I., Noronha Neto, C., \& Amorim, M. M. R. (2010). Indução do trabalho de parto: conceitos e particularidades. Femina.

Tahseen, S., \& Griffiths, M. (2010). Vaginal birth after two caesarean sections (VBAC-2) - a systematic review with meta-analysis of success rate and adverse outcomes of VBAC-2 versus VBAC-1 and repeat (third) caesarean sections. BJOG: An International Journal of Obstetrics \& Gynaecology, 117(1), 5-19.

Weidle, W. G., Medeiros, C. R. G., Grave, M. T. Q., \& Dal Bosco, S. M. (2014). Escolha da via de parto pela mulher: autonomia ou indução?. Cadernos Saúde Coletiva, 22(1), 46-53.

World Health Organization. (2015). WHO Statement on caesarean section rates. Reproductive health matters, 23(45), 149-150. https://doi.org/10.1016/j.rhm.2015.07.007.

Xie, R. H., Gaudet, L., Krewski, D., Graham, I. D., Walker, M. C., \& Wen, S. W. (2015). Higher cesarean delivery rates are as sociated with higher infant mortality rates in industrialized countries. Birth, 42(1), 62-69. 